\title{
Acute perimyocarditis mimicking acute myocardial infarction in a 12-year-old boy with Duchenne muscular dystrophy
}

\author{
Ho Jung Choi, Hye Won Kwon*, Kyung Jin Oh*, Mi Kyoung Song \\ Department of Pediatrics, Seoul National University Hospital, Seoul, Korea
}

Differential diagnosis of chest pain in the pediatric population is important but can be challenging. A 12-year-old boy with Duchenne muscular dystrophy presented with chest pain, cardiac enzyme elevation, and convex ST elevations in the inferior leads with reciprocal ST depression in the anterior leads on electrocardiogram. Echocardiography on admission revealed normal left ventricular function. Suspecting acute myocardial infarction, we performed invasive coronary angiography, which revealed normal coronary arteries. A follow-up electrocardiogram showed an acute pericarditis pattern with concave ST elevations in most leads and PR depression, and follow-up echocardiography revealed global left ventricular dysfunction, suggestive of acute perimyocarditis. Ibuprofen was administered for acute pericarditis, and a continuous milrinone infusion was commenced for myocardial dysfunction. The chest pain improved by the next day, and the ST segment elevations normalized on day 4. Echocardiography on day 9 revealed improved left ventricular function. The patient was discharged on day 11 , and he is doing well without chest pain through 12 months of follow-up. The last electrocardiogram showed normal sinus rhythm without ST change. Differential diagnosis of acute myocardial infarction and acute perimyocarditis is important for proper treatment strategies and the different prognoses of these two conditions.

Key Words: case report; myocarditis; pericarditis; ST elevation myocardial infarction

Differential diagnosis of chest pain in the pediatric population is important but can be challenging, especially in patients at risk for cardiomyopathy. We present a case of acute pericarditis in a 12-year-old boy with Duchenne muscular dystrophy (DMD) whose initial electrocardiogram (ECG) and cardiac enzyme levels were suggestive of ST-elevation myocardial infarction.

\section{CASE REPORT}

This work complies with the ethical standards of the relevant national guidelines on human medical regulations and with the Helsinki Declaration of 1975, as revised in 2008, and has been approved by the Institutional Review Board of Seoul National University Hospital (July 20th, 2020; IRB No. H-2007-099-1141). Because there is less than minimal risk to the patient,

\section{Case Report}

Received: March 8, 2021

Revised: April 30, 2021

Accepted: April 30, 2021

\section{Corresponding author}

Hye Won Kwon

Department of Thoracic and

Cardiovascular Surgery, Seoul

National University Hospital, 101

Daehak-ro, Jongno-gu, Seoul 03080, Korea

Tel: +82-2-2072-4306

Fax: +82-2-747-2471

E-mail:drhwkwon@gmail.com

${ }^{*}$ Current affiliation

Hye Won Kwon: Department of Thoracic and Cardiovascular Surgery, Seoul National University Hospital, Seoul, Korea

Kyung Jin Oh: Department of Pediatrics, Seoul Metropolitan Government-Seoul National University Boramae Medical Center, Seoul, Korea

Copyright (C) 2022 The Korean Society of Critical Care Medicine

This is an Open Access article distributed under the terms of Creative Attributions Non-Commercial License (https://creativecommons.org/li-censes/by-nc/4.0/) which permits unrestricted noncommercial use, distribution, and reproduction in any medium, provided the original work is properly cited. 
we submitted the waiver of informed consent to the IRB and obtained permission for waiver of consent.

A 12-year-old boy with DMD who is a wheelchair user presented to the emergency department with anterior chest pain in the sitting position that began 7 hours prior and radiated to the left back. The pain increased with inspiration. Four days previously, he had developed mild rhinorrhea and diarrhea without fever. Initial vital signs on arrival were as follows: blood pressure, 118/77 mm Hg; pulse rate, 79 beats per minute; respiratory rate, 18 breaths per minute; and body temperature, $36^{\circ} \mathrm{C}$. The last echocardiography performed 2 years prior showed normal cardiac function, and there was no abnormality in the last ECG.

A chest radiograph on arrival revealed the absence of cardiomegaly (Figure 1A). The initial ECG revealed convex ST elevation in leads II, III, aVF, and V6, with reciprocal ST depression in V1-3 (Figure 2A). Laboratory results revealed elevated troponin-I $(20.97 \mathrm{ng} / \mathrm{ml})$ and creatine kinase MB isoenzyme (147.9 ng/ml) and normal brain natriuretic peptide (BNP; $30 \mathrm{pg} / \mathrm{ml}$ )levels. Suspecting acute myocardial infarction, we performed invasive
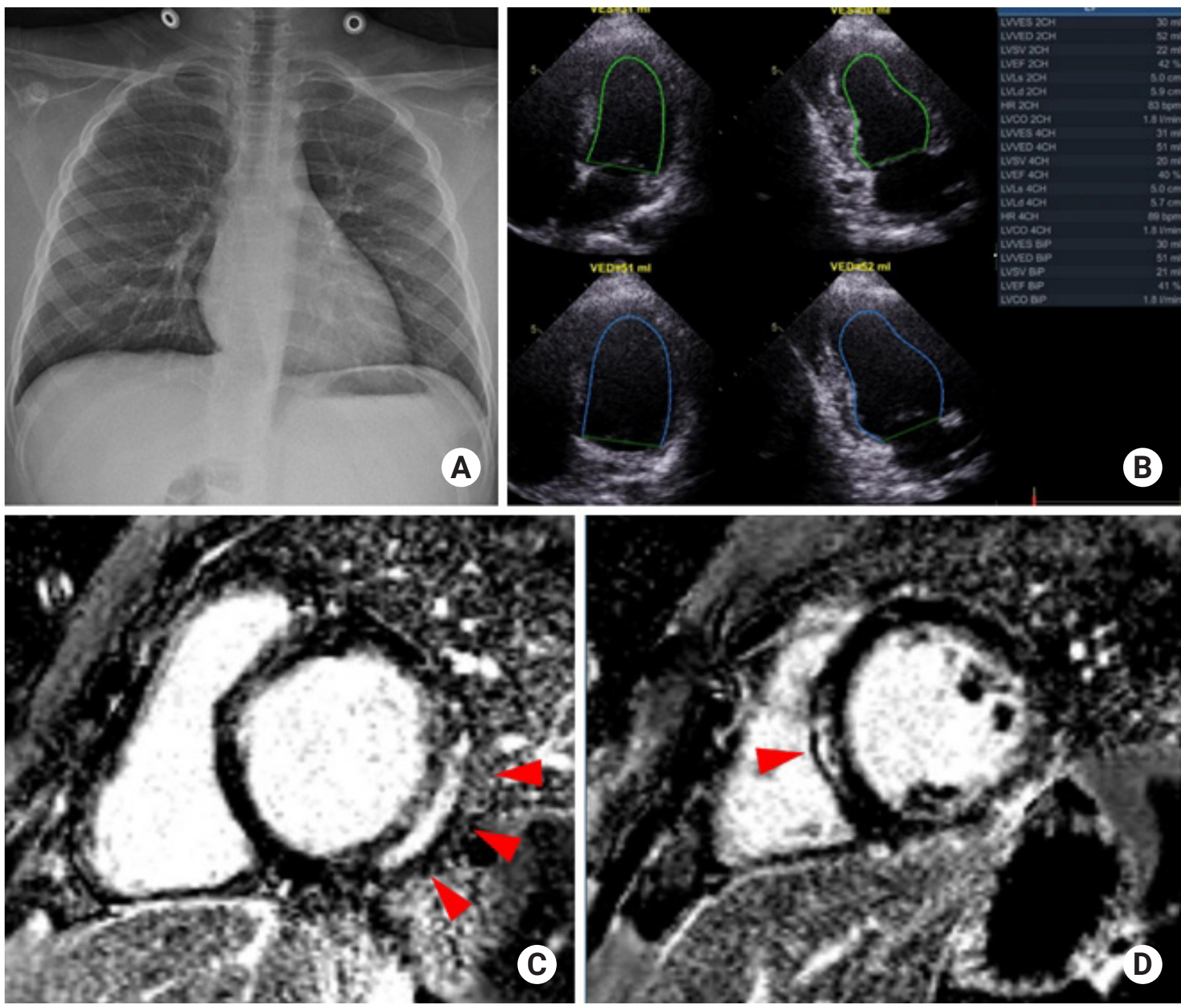

Figure 1. (A) Initial chest radiograph showing no cardiothoracic abnormalities. (B) Echocardiography performed on the second day of hospitalization showing mild global left ventricular dysfunction (ejection fraction, 41\%) without regional wall motion abnormalities. (C, D) Cardiac magnetic resonance imaging showing late gadolinium enhancement (arrowheads) in the subepicardium of the left ventricular inferolateral and mid-septal walls. 
coronary angiography, which revealed normal coronary arteries (Supplementary Video 1). After angiography, he was admitted to the pediatric intensive care unit for close monitoring of ECG changes. The echocardiograph on admission revealed normal left ventricular function (ejection fraction [EF], 66.2\%) without regional wall motion abnormalities.

Although the convex ST elevations and reciprocal changes on ECG remained aggravated at 3 hours post-admission (Figure 2B), an acute pericarditis pattern with concave ST elevations in most leads including V2-3 and PR depression developed 12 hours post-admission (Figure 2C). The next day, troponin-I level increased to $107.96 \mathrm{ng} / \mathrm{ml}$, and follow-up echocardiography revealed mild global left ventricular dysfunction (EF, 41\%) without visible pericardial thickening or effusion (Figure 1B, Supplementary Video 2). On the fifth day, the BNP level increased to $122 \mathrm{pg} / \mathrm{ml}$. Cardiac magnetic resonance imaging (MRI) showed mild global left ventricular dysfunction (EF, 48.1\%) with late gadolinium enhancement in the subepicardium of the left ventricular inferolateral and mid-septal walls (Figure $1 C$ and D). Respiratory viral polymerase chain reaction assay from a nasopharyngeal swab re- vealed a positive result for human coronavirus OC43. Tests for all other cardiotropic viruses including enterovirus, parvovirus B19, human herpes virus, influenza virus, coxsackievirus, respiratory syncytial virus, adenovirus, and Epstein-Barr virus were negative.

Suspecting acute viral pericarditis, we administered ibuprofen. Furthermore, a continuous milrinone infusion was commenced as the left ventricular function deteriorated rapidly within one day and cardiac MRI findings were suggestive of acute myocarditis. The chest pain improved by the next day, and the ST segment elevation normalized on the fourth day (Figure 2D). Echocardiography on the ninth day revealed improved left ventricular function (EF, 54\%), and intravenous milrinone was replaced with enalapril and carvedilol. The patient was discharged on the 11th day.

After 12 months of follow-up, he is doing well without chest pain. The last ECG showed normal sinus rhythm without pathologic ST-T changes, and a follow-up cardiac MRI performed at 4-month post-disease onset showed preserved left ventricular systolic function (EF, 54\%) without change of late gadolinium enhancement in the left ventricular inferolateral
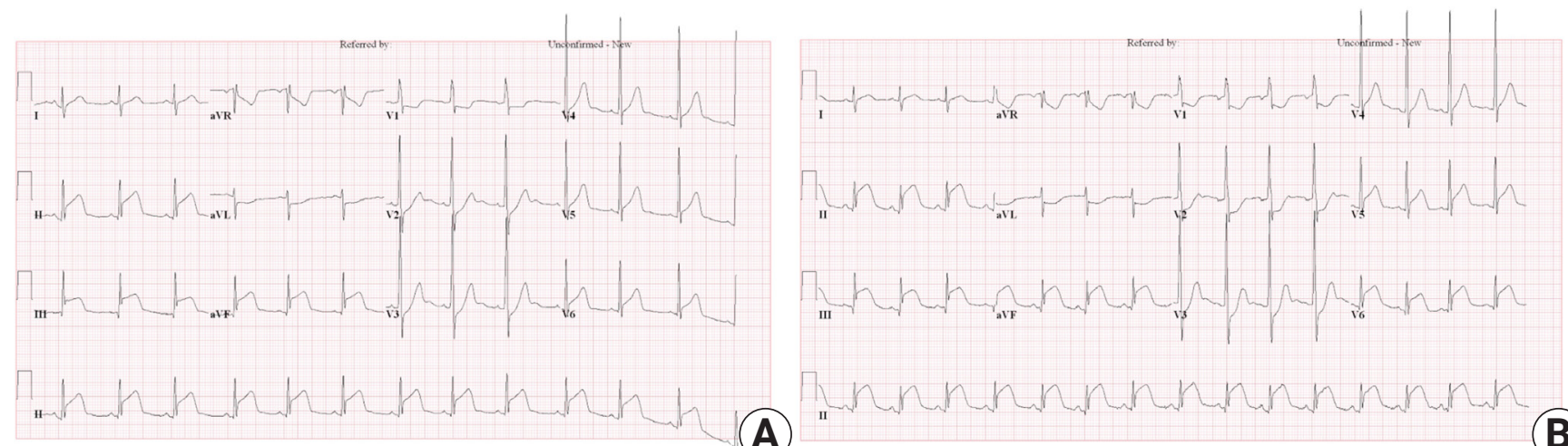

(A)
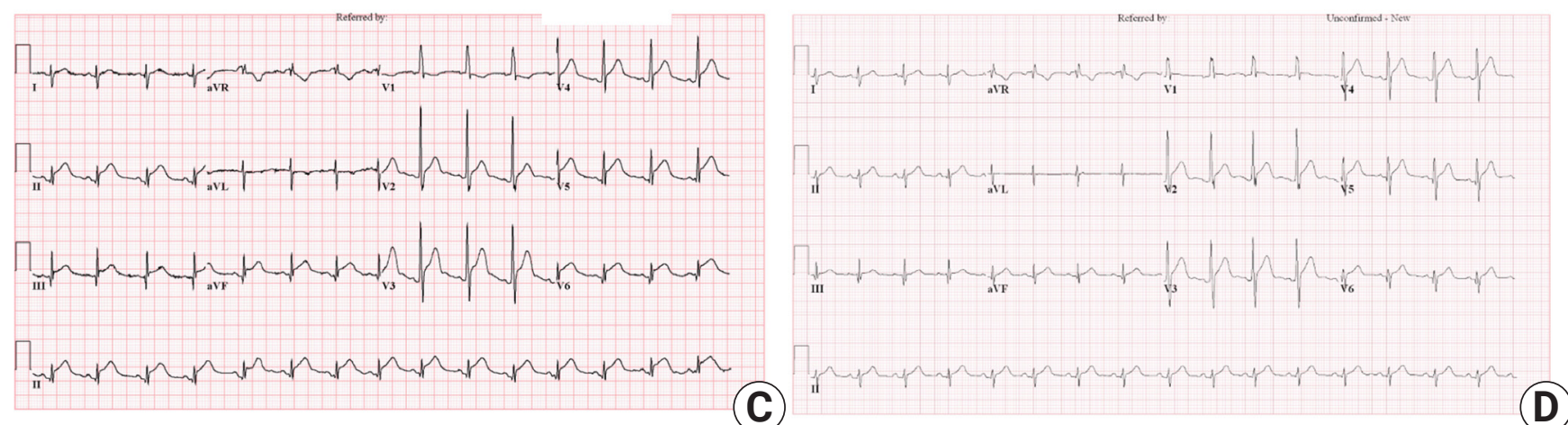

(C)

Figure 2. (A) Initial electrocardiogram showing ST elevations in the inferior leads and reciprocal ST depressions in the anterior leads. (B) Electrocardiogram, 3 hours post-admission, showing aggravated convex ST elevations and reciprocal changes. (C) Electrocardiogram, 12 hours post-admission, showing concave ST elevations in most leads and PR depression. (D) ST segment elevations normalized on the 4th day of hospitalization after ibuprofen treatment. aVR: lead augmented vector right; aVL: lead augmented vector left; aVF: lead augmented vector foot. 
wall, which was regarded as a sequela of previous myocarditis. The troponin-I and BNP levels decreased to $0.30 \mathrm{ng} / \mathrm{ml}$ and 93 $\mathrm{pg} / \mathrm{ml}$, respectively.

\section{DISCUSSION}

Typically, ST elevation in myocardial infarction has a convex morphology in the leads corresponding to the affected coronary territory, with concomitant reciprocal ST-segment depression in the opposing leads [1]. Conversely, in pericarditis, ST elevation with a concave morphology is usually present in most leads, and involvement of the atria is responsible for depression of the PR segment [1,2]. In the present case, the initial ECG pattern was typical of ST-elevation myocardial infarction, and the pattern changed to that of typical pericarditis within 12 hours of the onset of symptoms. This might be due to focal myocarditis that caused myocardial ischemia with accompanying pericarditis, causing repolarization abnormalities. Cases with evidence of new-onset focal or diffuse reduction in left ventricular function with elevated myocardial biomarkers and the presence of clinical criteria for acute pericarditis can be diagnosed as predominant myocarditis with pericardial involvement, or "perimyocarditis" [3].

Although the gold standard for diagnosis of myocarditis is endomyocardial biopsy, it is not recommended in specific circumstances, such as new-onset heart failure with accompanying hemodynamic compromise [4,5]. If one or more clinical symptoms are present with elevated cardiac biomarkers and characteristic features on echocardiography or cardiac MRI, myocarditis can be diagnosed $[6,7]$. We could not confirm whether coronavirus OC43 was the causative agent; however, it was highly suspected because the serology results for other cardiotropic viruses and the respiratory viral panel were negative. The pathogenesis of cardiac involvement associated with coronavirus remains unknown. Further investigation is warranted to ascertain whether the coronavirus can replicate and disseminate through the lymphatic system from the respiratory tract, or if the secondary immune response to viral infection could play a role in disease pathogenesis rather than the primary infection [8].

Additionally, considering the underlying disease of the patient, differential diagnosis with cardiomyopathy is important. Among children with DMD, the incidence of cardiomyopathy increases steadily in the teenage years [9]. The pathology and cardiac MRI pattern of cardiomyopathy in patients with dystrophinopathy classically produce subepicardial fibrosis of the inferolateral wall, as observed in some patients with viral myocarditis [10]. In our case, the cardiac dysfunction progressed rapidly and recovered quickly, suggesting a more acute course than that of cardiomyopathy. The common features of cardiomyopathy, such as ventricular dilatation, ventricular hypertrophy, or atrophy, were absent in this patient. Considering these factors, the diagnosis of acute perimyocarditis was more likely than that of cardiomyopathy, but this acute event could trigger earlier onset of cardiomyopathy in DMD.

In conclusion, patients with acute perimyocarditis can present with chest pain, focal ST-elevation, and significant elevation of cardiac enzymes, mimicking acute myocardial infarction. Differentiating between acute perimyocarditis and ST-elevation myocardial infarction is important because of differences in treatment strategies and prognoses. In the present case, the patient's young age and preceding viral illness favored a diagnosis of acute perimyocarditis, and the normal coronary angiography eliminated the possibility of acute coronary syndrome.

\section{CONFLICT OF INTEREST}

No potential conflict of interest relevant to this article was reported.

\section{ORCID}

Ho Jung Choi https://orcid.org/0000-0002-5256-5201

Hye Won Kwon https://orcid.org/0000-0002-4949-9688

Kyung Jin Oh https://orcid.org/0000-0003-3995-9705

Mi Kyoung Song https://orcid.org/0000-0002-0529-869X

\section{AUTHOR CONTRIBUTIONS}

Conceptualization: HWK. Data curation: HJC, KJO. Project administration: HWK. Writing-original draft: HJC. Writingreview \& editing: HWK, MKS.

\section{SUPPLEMENTARY MATERIALS}

Supplementary materials can be found via https://doi.org/ 10.4266/acc.2021.00290.

\section{REFERENCES}

1. de Bliek EC. ST elevation: differential diagnosis and caveats. A 
comprehensive review to help distinguish ST elevation myocardial infarction from nonischemic etiologies of ST elevation. Turk J Emerg Med 2018;18:1-10.

2. Deshpande A, Birnbaum Y. ST-segment elevation: distinguishing ST elevation myocardial infarction from ST elevation secondary to nonischemic etiologies. World J Cardiol 2014;6:106779.

3. Adler Y, Charron P, Imazio M, Badano L, Barón-Esquivias G, Bogaert J, et al. 2015 ESC Guidelines for the diagnosis and management of pericardial diseases: The Task Force for the Diagnosis and Management of Pericardial Diseases of the European Society of Cardiology (ESC)endorsed by: The European Association for Cardio-Thoracic Surgery (EACTS). Eur Heart J 2015;36:2921-64.

4. Kindermann I, Barth C, Mahfoud F, Ukena C, Lenski M, Yilmaz A, et al. Update on myocarditis. J Am Coll Cardiol 2012;59:779-92.

5. Cooper LT, Baughman KL, Feldman AM, Frustaci A, Jessup M, Kuhl U, et al. The role of endomyocardial biopsy in the management of cardiovascular disease: a scientific statement from the American Heart Association, the American College of
Cardiology, and the European Society of Cardiology Endorsed by the Heart Failure Society of America and the Heart Failure Association of the European Society of Cardiology. Eur Heart J 2007;28:3076-93.

6. Bozkurt B, Colvin M, Cook J, Cooper LT, Deswal A, Fonarow GC, et al. Current diagnostic and treatment strategies for specific dilated cardiomyopathies: a scientific statement from the American Heart Association. Circulation 2016;134:e579-646.

7. Sagar S, Liu PP, Cooper LT Jr. Myocarditis. Lancet 2012;379:73847.

8. Liu PP, Mason JW. Advances in the understanding of myocarditis. Circulation 2001;104:1076-82.

9. Nigro G, Comi LI, Politano L, Bain RJ. The incidence and evolution of cardiomyopathy in Duchenne muscular dystrophy. Int J Cardiol 1990;26:271-7.

10. Verhaert D, Richards K, Rafael-Fortney JA, Raman SV. Cardiac involvement in patients with muscular dystrophies: magnetic resonance imaging phenotype and genotypic considerations. Circ Cardiovasc Imaging 2011;4:67-76. 